\section{The Performance of Psoriatic Arthritis Screening Questionnaires in Patients with Psoriasis}

\section{To the Editor:}

Several screening questionnaires have been developed to identify patients with psoriatic arthritis (PsA) in the psoriasis population in dermatology and general practice settings $1,2,3,4,5$. However, the diagnosis of PsA using these questionnaires is a topic for debate, partly because of disease heterogeneity, and the complications from inconsistent performance results $6,7,8,9$

We aimed to evaluate the performance of the Psoriatic Arthritis Screening and Evaluation tool (PASE) ${ }^{1,2}$, the Psoriasis Epidemiology Screening Tool (PEST) $)^{2}$, the Toronto Psoriatic Arthritis Screen 2 (ToPAS $2)^{3}$, and the Early Arthritis for Psoriatic Patients (EARP) ${ }^{4}$ questionnaires in diagnosing PsA and to test the performance of the CONTEST questionnaire ${ }^{5}$ compared to the other existing tools.

Patients with psoriasis from the dermatology and combined rheumatology-dermatology clinics in one medical center completed the PASE, ToPAS 2, PEST, and EARP questionnaires in random sequence prior to rheumatologic evaluation. The PASE, ToPAS 2, PEST, and EARP questionnaires were translated from English to Hebrew by a professional translator after the appropriate institutions gave approval to use these questionnaires. The study was approved by the Ethics Board of The Lady Davis Carmel Medical Center in Haifa, Israel (0044-11-CMC).

A composite score was calculated by abstracting the score of each discriminatory item from the original tools that constitute the CONTEST questionnaire, because at the study start the CONTEST questionnaire had not been developed yet. Patients were assessed by a rheumatologist who was blinded to questionnaire results, and the diagnosis of PsA was determined according to the ClASsification for Psoriatic ARthritis (CASPAR) criteria $^{10}$.

The diagnostic accuracy of the questionnaires was assessed by calculating the receiver-operating characteristics (ROC) curves. The association between the questionnaires' performance and sex, level of education, family history of inflammatory arthritis, and systemic therapy was assessed with the $t$ test and Mann-Whitney U test or the ANOVA and Kruskal-Wallis test as appropriate.

From a total of 212 patients with psoriasis screened for inclusion, 208 patients who completed all the questionnaires were included in the study:
93 recruited from the combined rheumatology-dermatology clinic and 115 recruited from the primary care dermatology clinic. Of the 208 patients, 108 met the CASPAR criteria: 89 from the combined clinic and 19 from the dermatology clinic.

There was no significant difference in age, sex, level of education, type of psoriasis, family history of psoriasis or inflammatory arthritis, and rheumatoid factor positivity between the CASPAR-positive and CASPAR-negative groups. Patients with PsA had a higher C-reactive protein $(67.34 \%$ vs $6.25 \%, p<0.001)$ and were more likely to be treated with systemic therapy.

The area under the ROC curve (AUC) of all questionnaires was similar (Table 1 and Figure 1), with PEST being slightly higher, and yielding a positive predictive value of $94.4 \%$ and a negative predictive value of $80.9 \%$.

The sensitivities of PASE, ToPAS 2, PEST, EARP, and CONTEST were $57.9 \%, 60 \%, 79.4 \%, 78.0 \%$, and $70 \%$, respectively. The specificities were $93 \%, 93.2 \%, 94.9 \%, 91.8 \%$, and $91 \%$, respectively. When the cutoff point for the PASE questionnaire was changed from 47 to 34 , the sensitivity and specificity changed to $76.6 \%$ and $81.0 \%$, respectively.

There was no correlation between the performance of the questionnaires and age, sex, education level, or family history of inflammatory arthritis. Older patients had higher scores on the ToPAS 2 questionnaire, with no clinical relevance. CASPAR-positive patients who received systemic therapy obtained significantly higher scores on the PASE, PEST, EARP, and CONTEST questionnaires (PASE $\mathrm{p}=0.03, \operatorname{PEST} \mathrm{p}=0.003$, EARP $\mathrm{p}=0.01$, CONTEST $\mathrm{p}<0.0001)$, but not on the ToPAS 2 questionnaire $(\mathrm{p}=0.51)$.

This study is the first, to our knowledge, to present the data on all available screening questionnaires proposed and used in PsA including the CONTEST questionnaire. The CONTEST questionnaire had no additive diagnostic value to the other screening questionnaires, of which PEST had the highest sensitivity and specificity. The low sensitivity of ToPAS 2 compared to the other tools evaluated in the study was surprising in view of the questions and images depicting joint inflammation and dactylitis that should have increased its sensitivity.

We found a correlation between the use of systemic therapy and higher questionnaire scores. However, whether this suggests a correlation between disease severity and the scores or a confounder by severity is yet to be determined. Other confounders such as age, sex, family history of arthritis, and level of education had no effect of the performance of the questionnaires.

Table 1. Comparison of PASE, PEST, TOPAS, EARP, and CONTEST questionnaires.*

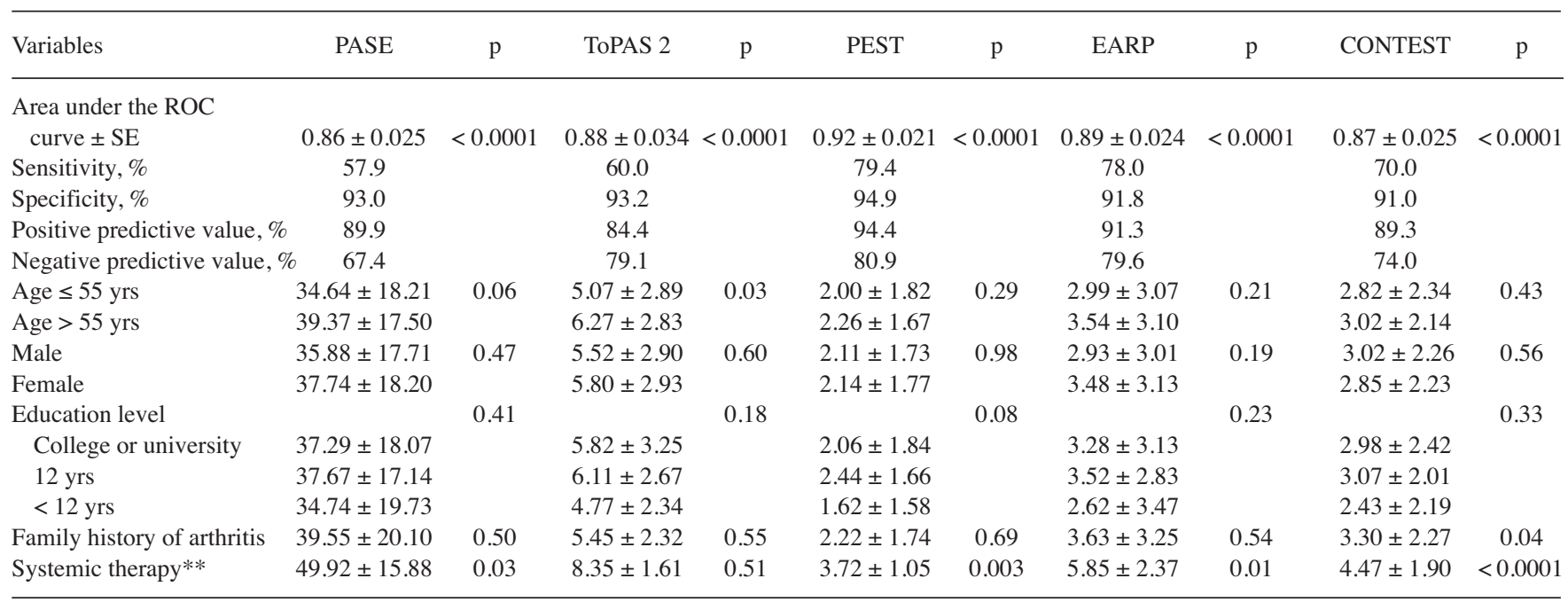

* Using the cutoff points of 47 for PASE, 8 for ToPAS 2, 3 for PEST, 3 for EARP, and 4 for the CONTEST. ** Only for CASPAR-positive patients. Values are mean \pm SE unless otherwise specified. ROC: receiver-operating characteristics (curves); SE: standard error; PASE: Psoriatic Arthritis Screening and Evaluation tool; PEST: the Psoriasis Epidemiology Screening Tool; ToPAS 2: the Toronto Psoriatic Arthritis Screen 2; EARP: Early Arthritis for Psoriatic Patients; CASPAR: ClASsification for Psoriatic ARthritis criteria. 


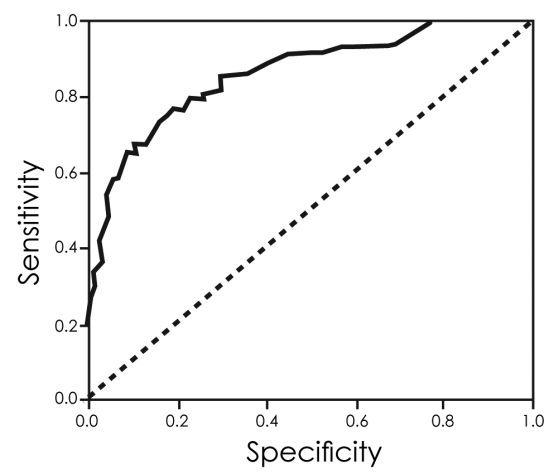

1b. TOPAS2 Questionnaire

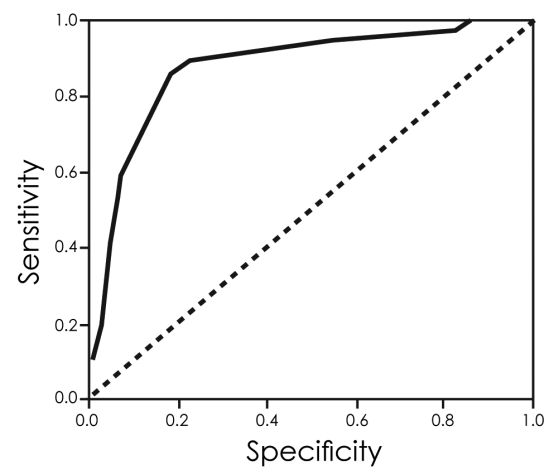

1d. EARP Questionnaire

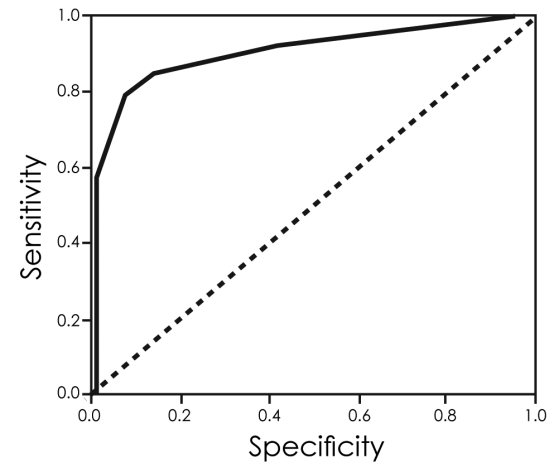

1c. PEST Questionnaire

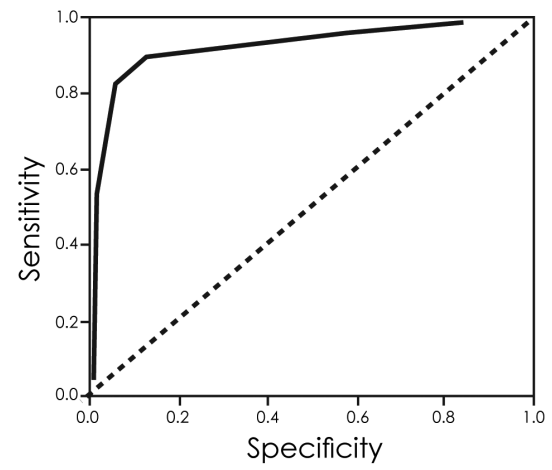

1e. CONTEST Questionnaire

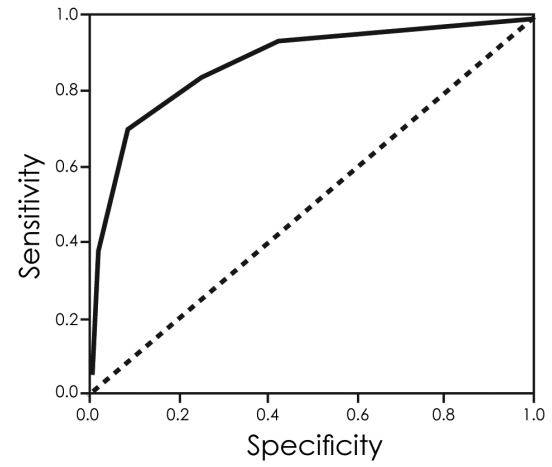

Figure 1. ROC curves for the 5 questionnaires. Cutoff points used for the questionnaires were 47 for PASE, 8 for ToPAS 2, 3 for PEST, 3 for EARP, and 4 for the CONTEST. ROC: receiver-operating characteristics (curves); PASE: Psoriatic Arthritis Screening and Evaluation tool; PEST: the Psoriasis Epidemiology Screening Tool; ToPAS 2: the Toronto Psoriatic Arthritis Screen 2; EARP: Early Arthritis for Psoriatic Patients.

Certain limitations of our study must be taken into consideration. Patients with mild psoriasis might have not been referred to a dermatology clinic, so the results of the performance of the questionnaires do not include this entire segment of the psoriasis population. Another limitation is the relatively small sample size. Moreover, because the CONTEST score was abstracted and not completed directly upon filling the form, the comparison of the CONTEST to the other existing questionnaires should be done with caution.

Our results indicate the ability of the screening questionnaires to exclude PsA in patients with psoriasis. However, they cannot serve as substitutes for clinical diagnosis, with the PEST questionnaire having the best tradeoff for PsA screening in a psoriasis population in the current study in primary and tertiary dermatology clinics.
AMIR HADDAD, MD, Rheumatology Unit, Carmel Medical Center; JOY FELD, MD, Rheumatology Unit, Carmel Medical Center; DEVY ZISMAN, MD, Rheumatology Unit, Carmel Medical Center, and The Ruth and Bruce Rappaport Faculty of Medicine, Technion, Haifa, Israel. Address correspondence to Dr. A. Haddad, Rheumatology Unit, Carmel Medical Center, 7 Michal St., Haifa 34362, Israel.

E-mail: haddadamir@yahoo.com.

\section{REFERENCES}

1. Husni ME, Meyer KH, Cohen DS, Mody E, Qureshi AA. The PASE questionnaire: pilot-testing a psoriatic arthritis screening and evaluation tool. J Am Acad Dermatol 2007;57:581-7. 
2. Dominguez PL, Husni ME, Holt EW, Holt EW, Tyler S, Qureshi AA. Validity, reliability, and sensitivity-to-change properties of the psoriatic arthritis screening and evaluation questionnaire. Arch Dermatol Res 2009;301:573-9.

3. Ibrahim GH, Buch MH, Lawson C, Waxman R, Helliwell PS. Evaluation of an existing screening tool for psoriatic arthritis in people with psoriasis and the development of a new instrument: the Psoriasis Epidemiology Screening Tool (PEST) questionnaire. Clin Exp Rheumatol 2009;27:469-74.

4. Tom BD, Chandran V, Farewell VT, Rosen CF, Gladman DD. Validation of the Toronto Psoriatic Arthritis Screen Version 2 (ToPAS 2). J Rheumatol 2015;42:841-6.

5. Tinazzi I, Adami S, Zanolin EM, Caimmi C, Confente S, Girolomoni G, et al. The early psoriatic arthritis screening questionnaire: a simple and fast method for the identification of arthritis in patients with psoriasis. Rheumatology 2012;51:2058-63.

6. Coates LC, Savage L, Waxman R, Moverley AR, Worthington S, Helliwell PS, et al. Comparison of screening questionnaires to identify psoriatic arthritis in a primary-care population: a cross-sectional study. Br J Dermatol 2016;175:542-8.
7. Coates LC, Aslam T, Al Balushi F, Burden AD, Burden-Teh E, Caperon AR et al. Comparison of three screening tools to detect psoriatic arthritis in patients with psoriasis (CONTEST study). Br J Dermatology 2013;168:802-7.

8. Mease PJ, Gladman DD, Helliwell P, Khraishi MM, Fuiman J, Bananis E, et al. Comparative performance of psoriatic arthritis screening tools in patients with psoriasis in European/North American dermatology clinics. J Am Acad Dermatol 2014; 71:649-55.

9. Mishra S, Kancharla H, Dogra S, Sharma A. Comparison of the Four Validated Psoriatic Arthritis Screening Tools in Diagnosing Psoriatic Arthritis in Patients with Psoriasis [COMPAQ Study]. Br J Dermatol 2017;176:765-70.

10. Taylor W, Gladman D, Helliwell P, Marchesoni A, Mease P, Mielants H; CASPAR Study Group. Classification criteria for psoriatic arthritis: development of new criteria from a large international study. Arthritis Rheum 2006;54:2665-73.

First Release November 1 2019; J Rheumatol 2019;46:12; doi:10.3899/jrheum.180939 